\title{
Propuesta de Clasificación Microclimática en función a la Vegetación predominante en la Cuenca del Rio Itaya, Loreto-Perú.
}

\section{Proposed Classification Microclimatic according to the predominant vegetation in the Itaya River Basin, Loreto-Perú.}

\author{
Diana Tello ${ }^{1}$, Marco Paredes ${ }^{2}$ y Marianela Cobos ${ }^{1}$
}

${ }^{1}$ Facultad de Ciencias e Ingeniería, Universidad Científica del Perú, Av. Abelardo Quiñones Km 2.5, Iquitos, Perú. Tel: 261088. diany tm 91@hotmail.com

${ }^{2}$ Servicio Nacional de Meteorología e Hidrología del Perú- SENAMHI

\begin{abstract}
Resumen
Clasificar un tipo de clima es un reto en selva media y baja, donde las variaciones solo se dan por influencia del crecimiento de las ciudades o los procesos de deforestación y por ello las metodologías de selección de climas no son suficientes para delimitar microclimas[1]. Por lo que; el objetivo principal del trabajo de investigación fue implementar una propuesta a nivel de microclima en función al tipo de vegetación predominante en la cuenca del río Itaya, el cual está flanqueado por dos principales ríos; Amazonas y Nanay. Se trabajaron con 47 estaciones climatológicas, que fueron ubicados dentro y fuera de la zona de estudio. Se utilizaron termohigrómetros digitales para el monitoreo de los puntos de muestreo. La metodología utilizada fue basado en Thornthwaite (1949)[2], para ello se dispuso de los valores mensuales de evapotranspiración, precipitación y temperaturas de la serie consecutiva de 35 años. Para realizar la propuesta de complementación a nivel de microclima se registraron variables como rango térmico, temperaturas mínimas y la humedad relativa. Determinándose un total de 19 microclimas. Los resultados muestran que los microclimas dependen del tipo de vegetación, en especial, de tipo aguajales en donde existe menor número de microclimas y son homogéneos, mientras que, en colinas predominan mayores oscilaciones térmicas heterogéneas y finalmente en zonas deforestadas existe una variedad de microclimas heterogéneos propios de su entorno. Por lo que este sistema de clasificación climática complementario a la metodología tradicional permite caracterizar microclimas en selva baja.
\end{abstract}

Palabras clave: microclima, propuesta, bosques, Itaya.

\begin{abstract}
Classify a type of climate is a challenge in medium and low forest where variations occur only under the influence of the growth of cities or deforestation processes and therefore the selection of climates methodologies are not sufficient to define microclimas [1]. So, the main objective of the research was to implement a proposed micro-level according to the predominant vegetation type in the Itaya River basin, which is flanked by two major rivers Amazon and Nanay. We worked with 47 weather stations were located inside and outside the study area. Thermohygrometers were used for monitoring digital sampling points. The methodology used was based on Thornthwaite (1949) [2], it was available for the monthly values of evapotranspiration, precipitation and temperatures of 35 consecutive series. To make the proposal to complement micro-level variables were recorded as thermal range, minimum temperature and relative humidity. Determining a total of 19 microclimates. The results show that the microclimates depend on the type of vegetation, especially aguajales type where there are fewer of microclimates and are homogeneous, while in hills dominate over thermal fluctuations and finally heterogeneous deforested areas there are a variety of microclimates heterogeneous to their environment. So this climate classification system complementary to the traditional methodology for characterizing micro-climates in the lowlands.
\end{abstract}

Keyword: microclimate, proposal,forests, Itaya 


\section{Introducción}

Una de las principales dificultades para la elaboración de Zonificación Ecológica y Económica a nivel meso o micro es la obtención de información climatológica básica, más aún, cuando se carece de estudios a nivel de cuenca o subcuenca. Esto se complica si existe el interés de determinar microunidades ambientales conocidas como "microclimas" donde se hace imprescindible información a mayor detalle de las variables; temperaturas, precipitación, humedad relativa, entre otras [1]. Por lo que, clasificar un tipo de clima es un reto en selva media y baja, donde las variaciones solo se dan por influencia del crecimiento de las ciudades o los procesos de deforestación y las metodologías de selección de climas no son suficientes para delimitar microclimas [2].

Se considera que las metodologías existentes para la clasificación climática no son apropiadas para zonas de selva baja donde las variaciones de los elementos climáticos no encajan en las categorías propuestas por la metodología de Thornthwaite, considerando a toda la selva como un clima súper húmedo, cálido, sin deficiencia de lluvias en el verano.

En ese sentido, el presente estudio propone una complementación a las metodologías tradicionales de clasificación climática para su utilización en delimitación de microclimas en selva media y baja teniendo en cuenta su relación con la vegetación predominante.

\section{Experimental}

\section{Área de Estudio}

La zona de estudio estuvo comprendida en el ámbito de la Cuenca del río Itaya, desde las cabeceras de cuenca hasta la desembocadura con el río Amazonas. La zona de estudio cubre un área total de $2618,38 \mathrm{~km}^{2}$ y un perímetro de $363,39 \mathrm{~km}$, está flanqueada por dos ríos de importancia: el Amazonas y el Nanay. Las coordenadas geográficas son; Latitud $03^{\circ} 34^{\prime} 00^{\prime \prime} \mathrm{S}$ a $04^{\circ} 38^{\prime} 45^{\prime \prime} \mathrm{S}$ y Longitud de $73^{\circ} 06^{\prime} 00^{\prime \prime} \mathrm{W}$ a $73^{\circ} 57^{\prime} 00^{\prime \prime} \mathrm{W}$.

\section{Muestreo}

La muestra estuvo conformada por 47 centros poblados ubicados en un área de $2618,38 \mathrm{Km}^{2}$ comprendida en la cuenca media y alta del río Itaya.

\section{Identificación de microclimas}

Para la identificación de los tipos climáticos se utilizó el método de [2], que tiene como fundamento el resultado final de las consideraciones sobre el Balance de Agua o balance hídrico. Los datos fueron obtenidos mediante dos fuentes: la primera proporcionada por el Servicio Nacional de Meteorología e Hidrología del Perú - SENAMHI que corresponden a 07 estaciones de los tipos Climatológica Ordinaria (CO) y la segunda fuente proveniente del Tropical Rainfall Measure Mission (TRMM) de quienes se obtuvieron 09 estaciones espaciales (fuera de la zona de estudio) y finalmente 31 estaciones temporales ubicados dentro de la zona de estudio, en donde se implementaron equipos de medición de temperatura y humedad por espacio de 45 días.

Para el cálculo de la evapotranspiración (ETP) se utilizó el método propuesto por [2]. Con las series de valores mensuales de ETP y precipitación se realizó el cómputo del balance hídrico mensual.

\section{Determinación de la vegetación predominante}

Para la descripción de las comunidades vegetales se utilizó el mapa de vegetación elaborado por el Instituto de Investigaciones de la Amazonía Peruana [3], desarrollado en el proyecto de "Microzonificación Ecológica Económica para el desarrollo sostenible del ámbito de la carretera lquitos-Nauta". Además se utilizó información geográfica existente del proyecto de "Macrounidades ambientales" elaborado por [4] el para constatar la vegetación predominante en algunas estaciones ubicadas alrededor de la zona de estudio.

\section{Formulación de la propuesta microclimática}

La propuesta microclimática en función al tipo de vegetación predominante, se formuló, considerando parámetros adicionales a las propuestas tradicionales tales como oscilación térmica, humedad relativa media y temperatura mínima.

\section{Instrumentos de recolección de datos}

La información de temperatura y humedad obtenida de los 47 puntos de control dentro de la cuenca hidrográfica del río Itaya recibió un control de calidad previo mediante la aplicación del análisis de consistencia de la información (doble masa).

Para la recolección de datos se empleó Termohigrómetro digital para el monitoreo de cada punto de muestreo. Cada estación permanente o temporal fue georeferenciada geográficamente usando el datum WSG84 y ploteado en una carta nacional digital usando el software Arcview 3.3.

\section{Resultados y discusión}

Se han identificado un total de 19 microclimas (Tabla 01). Estos resultados han permitido tener información detallada para ser utilizada en los estudios de zonificación económica. 
En esta nueva clasificación es notoria la relación en etapas iniciales con el proceso de deforestación severa que muchas veces modifican los climas, siendo afectados por factores locales (microclima). Por el contrario [4] a través del Proyecto BIODAMAZ realizó una clasificación climática para la zona de estudio, basándose en la metodología de Thornthwaite (1949) teniendo como resultado dos tipos de clima: B1 y B3 (húmedo y muy húmedo, cálido y sin deficiencia hídrica). De igual manera [5] presentaron un estudio de clasificación climática donde los criterios del sistema de clasificación de climas de Thornthwaite que está inspirado en las necesidades hidrológicas y agrícolas, antes que, en consideraciones puramente climatológicas, les permitió identificar un total de cinco tipos climáticos, cubriéndose toda la Provincia de Tocache, determinando que en todos los meses se presentan excedentes de humedad.

Posteriormente [6] utilizaron la misma metodología para la propuesta de ZEE en la zona de Bellavista Mazán, obteniéndose los tipos de clima para Puerto Almendras de B1 y para lquitos de C2, mostrándose algunos indicios de cambio, pero con características globales, aunque los sectores se traslapan, se tenían resultados un poco diferentes guardando semejanza con algunos microclimas identificados en el presente estudio. Y [7], elaboró una clasificación de las zonas de vida del mundo, en la que relaciona en forma cuantitativa la relación que existe en el orden natural entre los factores principales del clima y la vegetación como son; la biotemperatura, la precipitación y la humedad ambiental, como factores climáticos fundamentales.

\section{VEGETACIÓN PREDOMINANTES EN LA CUENCA DEL RÍO ITAYA}

La vegetación predominante estuvo determinado por 3 tipos:

\section{- Áreas Intervenidas con Actividades Antropogénicas}

Son zonas intervenidas a gran escala por la acción humana, generalmente muy utilizada para despejar grandes áreas de cobertura vegetal y están asociadas en la construcción de caminos, carreteras, centros poblados, ciudades y otras actividades, es muy dañina para el medio ambiente. La gran cantidad de dióxido de carbono (CO2) desprendida contribuye al efecto invernadero

La pérdida de cobertura vegetal o arbórea, destruye hábitats, acelera la erosión y multiplica la carga de sedimentos de los ríos, haciendo que las inundaciones estacionales sean mucho más graves.
Tabla 1: Microclimas identificados de acuerdo a la nueva propuesta

\begin{tabular}{|c|c|c|}
\hline $\mathrm{N}^{0}$ & Tipo de Microclima & $\begin{array}{c}\text { Propuesta } \\
\text { Complementaria }\end{array}$ \\
\hline & Clima Moderadamente húmedo, & \\
\hline 1 & $\begin{array}{l}\text { Ligeramente amplio, Aire húmedo con } \\
\text { Mañanas cálidas }\end{array}$ & B2LaHMc \\
\hline & Clima Ligeramente húmedo, Ligeramente & \\
\hline 2 & $\begin{array}{l}\text { amplio, Ligeramente húmedo con Mañanas } \\
\text { cálidas }\end{array}$ & B1LaLhMc \\
\hline & Clima Semi- húmedo con Amplio rango & \\
\hline 3 & $\begin{array}{l}\text { térmico, Ligeramente húmedo con Mañanas } \\
\text { cálidas }\end{array}$ & C2AmLhMc \\
\hline & Clima Semi- húmedo con Amplio re & \\
\hline 4 & $\begin{array}{l}\text { térmico, Ligeramente húmedo y Mañanas } \\
\text { extremadamente cálidas }\end{array}$ & C2AmLhMx \\
\hline & Clima Ligeramente húmedo con Amplio & \\
\hline 5 & $\begin{array}{l}\text { rango térmico, Ligeramente húmedo y } \\
\text { Mañanas extremadamente cálidas }\end{array}$ & B1AmLhMx \\
\hline 6 & $\begin{array}{l}\text { Clima Semi- húmedo, Ligeramente amplio, } \\
\text { Ligeramente húmedo y Mañanas cálidas }\end{array}$ & C2LaLhMc \\
\hline 7 & $\begin{array}{l}\text { Clima Semi-húmedo, Ligeramente amplio, } \\
\text { Ligeramente húmedo y Mañanas } \\
\text { extremadamente cálidas }\end{array}$ & C2LaLhMx \\
\hline & Clima Ligeramente húmedo con Amplio & \\
\hline 8 & $\begin{array}{l}\text { rango térmico, Aire húmedo y Mañanas } \\
\text { extremadamente cálidas }\end{array}$ & B1AmHMx \\
\hline 9 & $\begin{array}{l}\text { Clima Moderadamente húmedo con Amplio } \\
\text { rango térmico, Aire húmedo y Mañanas } \\
\text { cálidas }\end{array}$ & $\mathrm{B} 2 \mathrm{AmHMc}$ \\
\hline & Clima Moderadamente húmedo con Amplio & \\
\hline 10 & $\begin{array}{l}\text { rango térmico, Ligeramente húmedo y } \\
\text { Mañanas cálidas }\end{array}$ & B2AmLhMc \\
\hline 11 & $\begin{array}{l}\text { Clima Moderamente húmedo, Ligeramente } \\
\text { amplio, Ligeramente húmedo y Mañanas } \\
\text { extremadamente cálidas }\end{array}$ & B2LaLhMx \\
\hline 12 & $\begin{array}{l}\text { Clima Moderadamente húmedo con Amplio } \\
\text { rango térmico, Ligeramente húmedo y } \\
\text { Mañanas extremadamente cálidas }\end{array}$ & B2AmLhMx \\
\hline 13 & $\begin{array}{l}\text { Clima Húmedo con Amplio rango térmico, } \\
\text { Ligeramente húmedo y Mañanas cálidas }\end{array}$ & B3AmLhMc \\
\hline 14 & $\begin{array}{l}\text { Clima Húmedo con Amplio rango térmico, } \\
\text { Aire húmedo y Mañanas cálidas }\end{array}$ & B3AmHMc \\
\hline 15 & $\begin{array}{l}\text { Clima Ligeramente húmedo, Ligeramente } \\
\text { amplio, Ligeramente húmedo con Mañanas } \\
\text { extremadamente cálidas }\end{array}$ & B1LaLhMx \\
\hline 16 & $\begin{array}{l}\text { Clima Moderamente húmedo,Súper ámplio, } \\
\text { Ligeramente húmedo y Mañanas cálidas }\end{array}$ & B2SaLhMc \\
\hline 17 & $\begin{array}{l}\text { Clima Moderamente húmedo con Amplio } \\
\text { rango térmico, Aire húmedo y Mañanas } \\
\text { extremadamente cálidas }\end{array}$ & B2AmHMx \\
\hline 18 & $\begin{array}{l}\text { Clima Ligeramente húmedo con Amplio } \\
\text { rango térmico, Ligeramente húmedo y } \\
\text { Mañanas cálidas }\end{array}$ & B1AmLhMc \\
\hline 19 & $\begin{array}{l}\text { Clima Húmedo, Ligeramente amplio, } \\
\text { Ligeramente húmedo con Mañanas } \\
\text { extremadamente cálidas }\end{array}$ & B3LaLhMx \\
\hline
\end{tabular}

A esto se une el sistema de "tumba y quema", muy utilizados por las comunidades para hacer sus chacras y que dejan sin protección los suelos con la 
consecuencia de degradación y empobrecimiento de los mismos. [8]

\section{- Bosques de Colinas}

La existencia de bosques de colinas hasta una pendiente del $5 \%$, incluye las terrazas bajas y medianas. Esta formación presenta distintos grados de disección, fueron formadas por acción tectónica, interviniendo en la última fase de su modelado el efecto erosivo de la precipitación pluvial, con suelos superficiales a moderadamente profundos. El bosque presenta una densa y exuberante vegetación, alberga una gran diversidad vegetal y animal, en sus diferentes formas de vida, siendo el componente arbóreo el que domina sobre las otras formas de vida vegetal. El dosel superior del bosque está conformado por árboles vigorosos que superan los $40 \mathrm{~m}$ de altura.[9]

La cobertura es cerrada, el sotobosque es denso, presenta buen drenaje, los suelos son arcillosos de color marrón y naranja, de textura fina, lo atraviesan pequeñas quebradas, es frecuente encontrar los denominados supay chacras dominado por la especie Cordianodosa "Pucacuro Caspi" [10].

\section{- $\quad$ Palmerales de Mauritia flexuosa (aguajales)}

Se conoce como aguajales a las áreas pantanosas, que se encuentran permanentemente inundadas, donde crece la palmera aguaje (Mauritia flexuosa), incluyen aguajal denso y mixto. Se halla en las depresiones de los bosques de tahuampa y planicie de bajial o llanura, expuestos a la inundación periódico-estacional por agua negra o de mezcla. Presenta niveles freáticos muy altos, con drenaje y escorrentía lenta. Generalmente crecen sobre suelos de arena hidrometamórfica o espodozoles, con gruesas capas de humus que escurren agua negra [11]. Tiene una cobertura semi-cerrada, debido al diseño costillado-palmeada de las hojas de aguaje, con una altura máxima de dosel de $25 \mathrm{~m}$ y palmas emergentes de hasta $30 \mathrm{~m}$.[10]. Los aguajales abarcan unas 700 mil hectáreas de los 3,5 millones de hectáreas de bosque pantanoso [12]

\section{Relación de la Vegetación Predominante con los microclimas existentes en la Cuenca del Río Itaya}

Las zonas deforestadas 0 intervenidas con actividades antropogenicas tienen una característica particular que se encuentra afectada por los factores locales: como crecimiento poblacional, carreteras, poca densidad de vegetación, viviendas, que hacen que se manifiesten en variados elementos climáticos, es la zona donde podemos encontrar una gran variedad de microclimas. En la Fig. 01 se observa que en las áreas intervenidas el microclima C2AmLhMx (clima semi húmedo con amplio rango térmico, ligeramente húmedo [1] y mañanas extremadamente cálidas) se encuentran presente con mayor frecuencia en 6 estaciones climatológicas establecidas en diferentes centros poblados a diferencia de los microclimas (B2LaHMc, B1LaLhMc, C2LaLhMc,B1AmHMx, B2AmHMc, B3AmHMc, B2SaLhMc, B2AmHMx) que se encuentran con menor frecuencia.

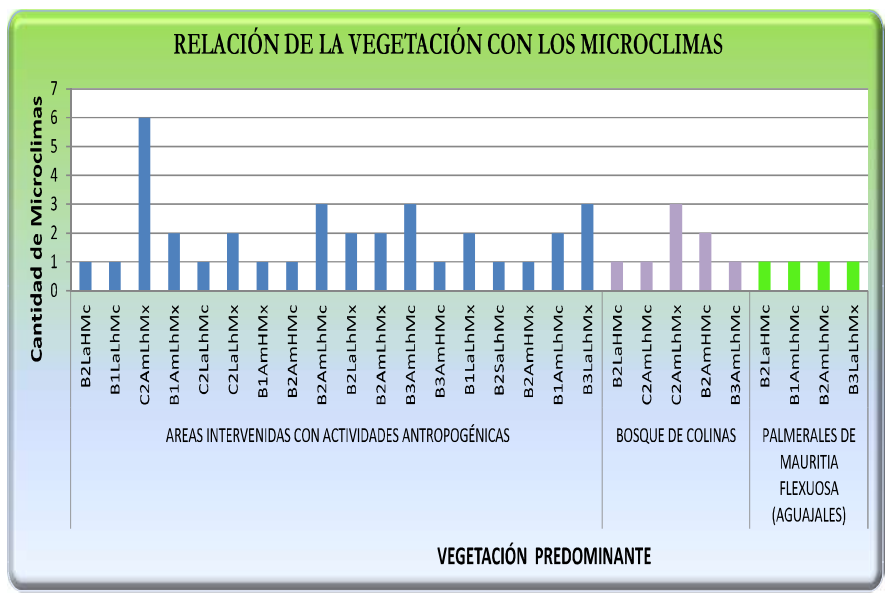

Fig. 1: Relación de microclimas de acuerdo al tipo de vegetación predominante

El Diagnostico del Cambio Climático de la Región Loreto [8] hace una referencia que el complejo de bosques de la Amazonía está íntimamente conectado al clima mundial. En principio, influye sobre el clima actuando como un gigantesco consumidor de calor cerca de la tierra, absorbiendo la mitad de la energía solar que le llega a través de la evaporación del agua de su follaje. En segundo lugar es una reserva amplia y relativamente sensible del carbono que se libera a la atmosfera a través de la deforestación, la sequía y el fuego, contribuyendo a la acumulación atmosférica de gases que atrapan calor y que son las causas del calentamiento global. En tercer lugar, el agua que drena de estos bosques hacia el océano atlántico constituye del 15 al $20 \%$ de la descarga total mundial de agua dulce fluvial y podría ser suficiente para influir sobre algunas de las grandes corrientes oceánicas que son, en sí, importantes reguladoras del sistema climático global

Bajo este contexto, mediante esta nueva propuesta de clasificación microclimática se podrá definir los diferentes climas que hay en selva baja a escala de 
microclima. Ya que tiene diferentes variables como rango térmico, humedad relativa media $y$ temperatura mínima, todos estos parámetros basados según el Panel Intergubernamental de Cambio Climático. [13]

\section{Conclusiones}

Se han identificado 19 tipos de microclimas en la cuenca del rio Itaya, las cuales son: LaHMc, LaLhMc, AmLhMc, AmLhMx, AmLhMx, LaLhMc,LaLhMx, AmHMx, AmHMc, AmLhMc, LaLhMx, AmLhMx, AmLhMc, AmHMc, LaLhMx, SaLhMc, AmHMx, AmLhMc, LaLhMx.

La relación que existe entre los tipos de bosques y los microclimas identificados es que los bosques deforestados están influenciados por factores locales y por lo tanto tiene diversos tipos de microclimas. Mientras, los bosques de aguajales y de colinas se caracterizan por tener amplio rango térmico.

Se elaboró un sistema de clasificación climática complementario a la metodología tradicional basada en el rango térmico, humedad relativa y temperatura mínima

\section{AGRADECIMIENTOS}

Al Servicio Nacional de Meteorología e Hidrología del Perú (SENAMHI) por la información suficiente para la realización de la investigación y al Instituto de Investigaciones de la Amazonía Peruana (IIAP) por brindar las facilidades para la obtención de información bibliográfica

\section{Referencias}

[1] IIAP. (2011). Zonificación Ecológica y Económica de la Provincia de Satipo. IquitosPerú, 126 pp.

[2] THORNTHWAITE (1949). Clasificación de Thornthwaite. Disponible en: ocw.upm.es/ingeniería/Thornthwaiteclasificac ionclimatica.pdf
[3] IIAP (2012). "Proyecto de Microzonificación ecológica económica para el desarrollo sostenible del ámbito de la carretera lquitosNauta". Mapa de vegetación.

[4] IIAP-BIODAMAZ (2007). Mapa de macrounidades ambientales.

[5] VARGAS y MACO (2006). Estudio temáticos para la zonificación ecológica y económica de la provincia de Tocache. Convenio entre el IIAP y la Municipalidad Provincial de Tocache. Iquitos-Perú, 33 pp.

[6] ZUMAETA et al. (2009). Plan de ordenamiento Territorial Bellavista Mazán. Proyecto Binancional Desarrollo Integral de la Cuenca del Rio Putumayo.

[7] HOLDRIDGE, L.R. (1967). Life Zone Ecology. Tropical Science Center. San José de Costa Rica.

[8] GOREL (2010). Diagnóstico del Cambio Climático de la Región Loreto. Iquitos. 122 p.

[9] VELASQUEZ RIOS, H. (1991). Eficiencia del inventario forestal del bosque húmedo tropical de San Antonio - Rio Itaya. Tesis Ingeniero Forestal, Univ. Nacional de la Amazonía Peruana, lquitos, $73 p$.

[10] COBOS, M. (2011). Propuesta técnica para la solicitud de otorgamiento de concesión para conservación. Comunidad ribereña de Luz del Oriente, cuenca alta del Río Itaya, Distrito de San Juan Bautista, provincia de Maynas-Región Loreto.

[11] ENCARNACIÓN, F. (1993). El bosque y las formaciones vegetales en la llanura amazónica del Perú. Alma Mater 6:95-114.

[12] MALLEUX, J. 1975. Mapa Forestal del Perú (Memoria Explicativa). Departamento de Manejo Forestal, Universidad Nacional Agraria La Molina. Lima, Perú. 161 pp.

[13] IPCC (2007). Cambio Climático 2007. Disponible en:

http://www.ipcc.ch/pdf/assessmentreport/ar4/syr/ar4_syr_sp.pdf

E-mail: diany_tm_91@hotmail.com 